\title{
Risk of Death due to Hepatocellular Carcinoma among Drinkers and Ex-drinkers. Univariate Analysis of JACC Study Data
}

\author{
ITSURO OGIMOTO, AKIRA SHIBATA, YOUICHI KUROZAWA*, TAKAYUKI NOSE*, \\ TAKESUMI YOSHIMURA**, HIROSHI SUZUKI ${ }^{\dagger}, N^{*}$ BUO IWAI", RITSU SAKATA, \\ YUKI FUJITA, SHOKO ICHIKAWA, KATSUHIRO FUKUDA, \\ AKIKO TAMAKOSHI ${ }^{\S}$ AND THE JACC STUDY GROUP§§ \\ Department of Public Health, Kurume University School of Medicine, Kurume 830-0011, \\ * Division of Health Administration and Promotion, Department of Social Medicine, Faculty of \\ Medicine, Tottori University, Yonago 683-8503, \\ ** Department of Clinical Epidemiology, Institute of Industrial and Ecological Sciences, University \\ of Occupational and Environmental Health, Kitakyushu 807-8555, \\ † Department of Infectious Disease Control and International Medicine, Niigata University \\ Graduate School of Medical and Dental Sciences, Niigata 951-8510, \\ ¥ Chugoku Occupational Health Association, Tottori 680-0942, \\ $\S$ Department of Preventive Medicine/Biostatistics and Medical Decision Making, Nagoya University \\ Graduate School of Medicine, Nagoya 466-8550, Japan. \\ $\S \S$ See acknowledgments for present and the past investigators involved in the JACC Study.
}

\begin{abstract}
Summary: Hazard ratios (HR) of death due to hepatocellular carcinoma (HCC) were analyzed by gender and age strata (40-59 and 60-79) among drinkers and ex-drinkers in 66,974 eligible subjects from a a large cohort of male and female subjects aged 40-79 years, based on information about several drinking related characteristics. The HR of dying from HCC for ex-drinkers was 4 to 8 times higher than for those who had never consumed alcohol at the baseline survey. When the subjects were restricted to those without history of liver disease (LD), the HR was still high for ex-drinkers among younger males, though the difference was not statistically significant. It appeared that the earlier drinking habits were established, the higher the HR, especially for younger males without LD. Among total current drinkers, the amount ingested per occasion and the cumulative amount ingested at the baseline did not show significantly increased HRs. Among subjects without LD, larger amounts ingested per occasion and larger cumulative amount seemed to have higher HRs in older male current drinkers. Frequent drinking and later age (50 to 79) at cessation of drinking were associated with higher HRs among both genders and both age strata. After restricting the analysis to subjects without LD, many of these increased HRs remained among males. The results suggested that the association between alcohol drinking history and HR of HCC differs depending on the presence of LD. Major confounders other than age and gender associated with both drinking and HCC, e.g. smoking, hepatitis virus infection, or history of diabetes, were not considered in this analysis, and the observed associations might be confounded by any of these factors. To clarify the net association between alcohol drinking and $\mathrm{HCC}$, further analysis is needed to control potential confounders, including past history of liver disease, and to consider probable effect modifiers.
\end{abstract}

Key words JACC Study, alcohol drinking, death from hepatocellular carcinoma, Japanese

Received for publication December 22, 2003

Mail and reprint requests to: Itsuro Ogimoto, Department of Public Health, Kurume University School of Medicine, 67 Asahi-machi, Kurume 830-0011, Japan. Tel: 0942-31-7553 Fax: 0942-31-7698 


\section{INTRODUCTION}

Alcohol drinking is considered to be a risk factor for occurrence of and death from hepatocellular carcinoma (HCC) [1-19]. Alcohol consumption can be evaluated from several aspects, e.g. current drinking status, drinking history, and habitual or cumulative drinking amount. Unfortunately, drinking habits are not stable and may vary greatly over time, and there is a large variation among persons in terms of age of initiation and cessation, or drinking amount. This has resulted in a variety of definitions being used in different studies. Generally, however, it has been suggested that status as a current drinker and status as a heavy drinker are risk factors for occurrence of and death from HCC. As a preparation for a multivariate analysis of the Japan Collaborative Cohort (JACC) Study data on drinking and smoking, a univariate analysis was performed separately for each survey item concerned with alcohol drinking history.

\section{MATERIALS AND METHODS}

\section{Subjects and Identification of death from HCC}

Baseline survey data from 110,792 cohort members in the JACC Study was used for this analysis. Full details of the methodology of the JACC Study were described elsewhere [20]. Briefly, 46,465 males and 64,327 females aged between 40 and 79 at registration were enrolled in the cohort from 1988 to 1990. Subjects were followed until the end of 1999. Residential and survival status were confirmed by searching official residential rosters for changes of address or death, and in cases of the death the death certificate was examined for cause and date of death, with permission from the Director-General of the Prime Minister's Office.

The end point of follow-up for the present analysis was death from HCC, coded as C22.0 in the International Classification of Diseases and Related Health Problems, 10th Revision (ICD-10).

Individuals who reported past history of liver cancer and subjects coded C22.9 (malignant neoplasms of the liver, not otherwise specified) were eliminated from the analysis to allow the presence or absence of HCC to be more accurately distinguished. Deaths from HCC within 2 years after registration in the JACC Study cohort were suspected to have been affected by HCC at or before the time of registration and they were also eliminated from the analysis.

\section{Questionnaire and data correction}

Informed consent was obtained for participation in the study, after which subjects were interviewed or filled out questionnaires by themselves. Information obtained was transformed into the JACC Study standard questionnaire format and submitted to the central office. A working group for cleaning up data reviewed the transformed dataset with regard to distribution and logical accuracy. Details of this process were described in a previous report [21].

Some differences in the questionnaires among collaborating institute may cause systematic gaps in data in the transformed dataset and differences in the number of subjects to be analyzed by variable. To avoid these differences, the analyzed subjects were restricted to persons from collaborating institutes whose surveys contained almost the same questions concerning smoking and drinking.

\section{Drinking history}

Baseline information obtained about alcohol drinking history consisted of (1) drinking habits either never, ex- or current-drinker for all subjects, (2) drinking frequency in 4 grades for ex- and current-drinkers, (3) age at start of drinking, change in amount of alcohol consumed, type of alcoholic beverage and average amount consumed per occasion for current-drinkers, and (4) age at cessation of drinking and years since cessation of drinking for exdrinkers. Amount consumed was measured in terms of sake (a popular Japanese alcoholic beverage) and converted into number of Japanese 'gou' units $(0.180$ liters). Cumulative amount of alcohol consumed until the baseline (cumulative amount) was then calculated as the product of the number of 'gou' and the number of years of drinking obtained by subtracting age at start of drinking from age at the baseline, and expressed in 'gou-years'. Names of variables used for analysis basically follow the names used in the official progress report of the JACC Study published in March, 1996 [22].

\section{Statistical analysis}

The primary objective of this study was to observe the outward effect of single drinking characteristics on the hazard ratio (HR) of death from HCC. Therefore, in this analysis, only gender, age, past history of liver disease (LD) and collaborating institutes were considered as confounders [21].

A Cox proportional hazards model based on stratification by institute (SAS PHREG procedure 
with STRATA statement [23]) was used to obtain the HR of dying due to $\mathrm{HCC}$ in association with drinking habits, age at start of drinking, frequency, change in amount of alcohol consumed, drinking amount and age at cessation of drinking. A combined variable called "summary drinking history" consisting of drinking habits, drinking frequency and age at cessation of drinking was defined in order to estimate HR from several characteristics of personal drinking history simultaneously.

Analysis was done separately by gender, age (4059: younger, 60-79: older), and LD. Subjects with unknown LD were omitted from the analysis.

This study was approved by the Ethics Committee of the Kurume University School of Medicine.

\section{RESULT}

\section{Number of analyzed subjects}

After eliminating the subjects who had had history of liver cancer at the baseline and those who died of C22.9 during the follow-up period or died of C22.0 within two years from registration in the study, 110,688 subjects were left and 401 deaths with C22.0 were observed.

Subsequently, 29,435 subjects from collaborating institutes whose datasets had large data gaps, 10,743 subjects with unknown LD and 3,536 who didn't respond to questions about drinking habits were omitted, and as a result the number of subjects for analysis decreased to 66,974 (Male: 28,343, Female: 38,631 ) including 184 HCC (Table 1). Distribution of the subjects by characteristic is presented along with estimated HR (Table 2-7).

\section{Hazard ratios by drinking characteristics}

Results are presented for two types of datasets, i.e. one for total subjects that consists of both those with LD and without LD (total subjects), and another for restricted subjects that consists of only those without LD. Among the 66,974 total subjects, those who were ex-drinkers showed elevated HRs of 4 to 8 compared to those who had never drunk alcohol at the baseline, and those ratios were statistically significant except in younger females. Later age (30 or more) at start of drinking had significantly lower HRs and an inverse trend was suggested for the age at start of drinking for total younger males (Table 2).

After subjects were restricted to those without LD, HRs were still high for ex-drinkers among younger males though this difference was not statistically significant (Table 3).

Among total current drinkers, HRs for drinking amount and cumulative amount at the baseline were not significantly increased (Table 4). In restricted subjects without LD, larger amount consumed and larger cumulative amount had higher HRs in younger males (Table 5).

Among total male ex-drinkers, frequent drinking and later age at cessation of drinking presented higher HRs in each strata (Table 6). After restricting to subjects without LD, many of these increased HR remained among males (Table 7). Years since cessation of drinking showed HR 22 times higher than never drinkers for younger males at 6 to 10 years since cessation of drinking (Table 7), besides the HR was 3 to 4 among subjects with LD. (data was not

TABLE 1.

Age and sex distribution of subjects analyzed by past history of liver disease

\begin{tabular}{|c|c|c|c|c|c|c|c|c|c|}
\hline \multirow{2}{*}{$\begin{array}{c}\text { Age at } \\
\text { registration }\end{array}$} & \multicolumn{3}{|c|}{ Without history } & \multicolumn{3}{|c|}{ With history } & \multicolumn{3}{|c|}{ Total } \\
\hline & Male & Female & Both & Male & Female & Both & Male & Female & Both \\
\hline $40-44$ & 4,233 & 5,240 & 9,473 & 2 & 0 & 2 & 4,235 & 5,240 & 9,475 \\
\hline $45-49$ & 3,919 & 5,245 & 9,164 & 3 & 2 & 5 & 3,922 & 5,247 & 9,169 \\
\hline $50-54$ & 3,895 & 5,604 & 9,499 & 11 & 3 & 14 & 3,906 & 5,607 & 9,513 \\
\hline $55-59$ & 4,628 & 6,426 & 11,054 & 24 & 8 & 32 & 4,652 & 6,434 & 11,086 \\
\hline $60-64$ & 4,915 & 6,385 & 11,300 & 37 & 16 & 53 & 4,952 & 6,401 & 11,353 \\
\hline $65-69$ & 3,053 & 4,833 & 7,886 & 21 & 15 & 36 & 3,074 & 4,848 & 7,922 \\
\hline $70-74$ & 2,181 & 2,922 & 5,103 & 22 & 9 & 31 & 2,203 & 2,931 & 5,134 \\
\hline $75-79$ & 1,394 & 1,917 & 3,311 & 5 & 6 & 11 & 1,399 & 1,923 & 3,322 \\
\hline Total & 28,218 & 38,572 & 66,790 & 125 & 59 & 184 & 28,343 & 38,631 & 66,974 \\
\hline
\end{tabular}


TABLE 2.

Hazard ratio of death from HCC by several characteristics in drinking history among total subjects

\begin{tabular}{|c|c|c|c|c|c|c|c|c|c|c|c|c|}
\hline \multirow[b]{3}{*}{ Characteristics } & \multicolumn{6}{|c|}{ Male } & \multicolumn{6}{|c|}{ Female } \\
\hline & \multicolumn{3}{|c|}{$40-59$} & \multicolumn{3}{|c|}{$60-79$} & \multicolumn{3}{|c|}{$40-59$} & \multicolumn{3}{|c|}{$60-79$} \\
\hline & $\begin{array}{c}\text { Number } \\
\text { of } \\
\text { subjects }\end{array}$ & $\begin{array}{l}\text { Hazard } \\
\text { Ratio }^{1)}\end{array}$ & $\begin{array}{c}95 \% \\
\text { confidence } \\
\text { interval }\end{array}$ & $\begin{array}{c}\text { Number } \\
\text { of } \\
\text { subjects }\end{array}$ & $\begin{array}{l}\text { Hazard } \\
\text { Ratio }^{1)}\end{array}$ & $\begin{array}{c}95 \% \\
\text { confidence } \\
\text { interval }\end{array}$ & $\begin{array}{c}\text { Number } \\
\text { of } \\
\text { subjects }\end{array}$ & $\begin{array}{l}\text { Hazard } \\
\text { Ratio }^{1)}\end{array}$ & $\begin{array}{c}95 \% \\
\text { confidence } \\
\text { interval }\end{array}$ & $\begin{array}{c}\text { Number } \\
\text { of } \\
\text { subjects }\end{array}$ & $\begin{array}{l}\text { Hazard } \\
\text { Ratio }^{1)}\end{array}$ & $\begin{array}{c}95 \% \\
\text { confidence } \\
\text { interval }\end{array}$ \\
\hline $\begin{array}{l}\text { Alcohol drinking habits } \\
\text { (Referent: never drinker) }\end{array}$ & 2,707 & 1.00 & & 2,550 & 1.00 & & 16,256 & 1.00 & & 12,816 & 1.00 & \\
\hline Ex-drinker & 592 & 8.11 & $3.17-20.77^{*}$ & 1,085 & 3.48 & $1.86-6.54^{*}$ & 335 & 3.85 & $0.48-30.93$ & 297 & 4.18 & $1.47-11.88^{*}$ \\
\hline Currently drinker & 13,416 & 0.65 & $0.27-1.52$ & 7,993 & 0.75 & $0.43-1.31$ & 5,937 & 0.23 & $0.03-1.80$ & 2,990 & 0.59 & $0.25-1.43$ \\
\hline \multicolumn{13}{|c|}{ Age at start of drinking (current drinkers) } \\
\hline (Referent: never drinker) & 2,707 & 1.00 & & 2,550 & 1.00 & & 16,256 & 1.00 & & 12,816 & 1.00 & \\
\hline Less than 20 & 813 & 1.43 & $0.37-5.57$ & 273 & 0.53 & $0.07-4.05$ & 59 & - & & 16 & - & \\
\hline 20 to less than 30 & 6,109 & 0.82 & $0.32-2.11$ & 2,698 & 0.91 & $0.47-1.77$ & 892 & 1.52 & $0.19-11.91$ & 235 & 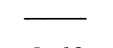 & \\
\hline 30 or more & 4,729 & 0.17 & $0.04-0.83^{*}$ & 3,864 & 0.66 & $0.35-1.27$ & 2,705 & - & & 1,406 & 0.63 & $0.19-2.08$ \\
\hline \multicolumn{13}{|c|}{ Drinking frequency (current drinkers) } \\
\hline (Referent: never drinker) & 2,707 & 1.00 & & 2,550 & 1.00 & & 16,256 & 1.00 & & 12,816 & 1.00 & \\
\hline Less than 1 times a week & 854 & 0.41 & $0.05-3.68$ & 398 & 0.73 & $0.18-2.93$ & 1,735 & - & & 638 & 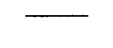 & \\
\hline 1 or 2 times a week & 1,243 & 1.27 & $0.37-4.35$ & 599 & 1.35 & $0.53-3.44$ & 1,626 & - & & 725 & $\longrightarrow$ & \\
\hline 3 or 4 times a week & 1,932 & 0.65 & $0.17-2.55$ & 851 & 0.78 & $0.29-2.13$ & 1,154 & $\overline{\rho^{\prime}}$ & & 556 & - & \\
\hline Almost everyday & 9,193 & 0.59 & $0.24-1.49$ & 5,931 & 0.63 & $0.34-1.15$ & 1,232 & 0.94 & $0.12-7.49$ & 863 & 0.69 & $0.16-2.93$ \\
\hline \multicolumn{13}{|c|}{ Change in amount of alcohol consumed (current drinkers) } \\
\hline (Referent: effort to decrease) & 1,186 & 1.00 & & 1,169 & 1.00 & & 333 & 1.00 & & 202 & 1.00 & \\
\hline Decrease & 3,301 & 0.71 & $0.24-2.11$ & 3,125 & 0.93 & $0.45-1.90$ & 1,115 & - & & 707 & 0.94 & $0.10-9.08$ \\
\hline No change & 5,941 & 0.18 & $0.05-0.66^{*}$ & 2,702 & 0.26 & $0.10-0.71 *$ & 2,798 & - & & 1,281 & 0.16 & $0.01-2.61$ \\
\hline Increase & 2,180 & 0.14 & $0.02-1.16$ & 531 & 0.48 & $0.11-2.15$ & 817 & $\longrightarrow$ & & 197 & & \\
\hline
\end{tabular}

1) Result from SAS PHREG procedure with STRATA statement on variable concerning with collaborating institute.

${ }^{*} \mathrm{p}<0.05, \wedge \mathrm{p}<0.10$

HR is not available because of thin data. 
TABLE 3.

Hazard ratio of death from HCC by characteristics in drinking history among restricted subjects without past history of liver disease

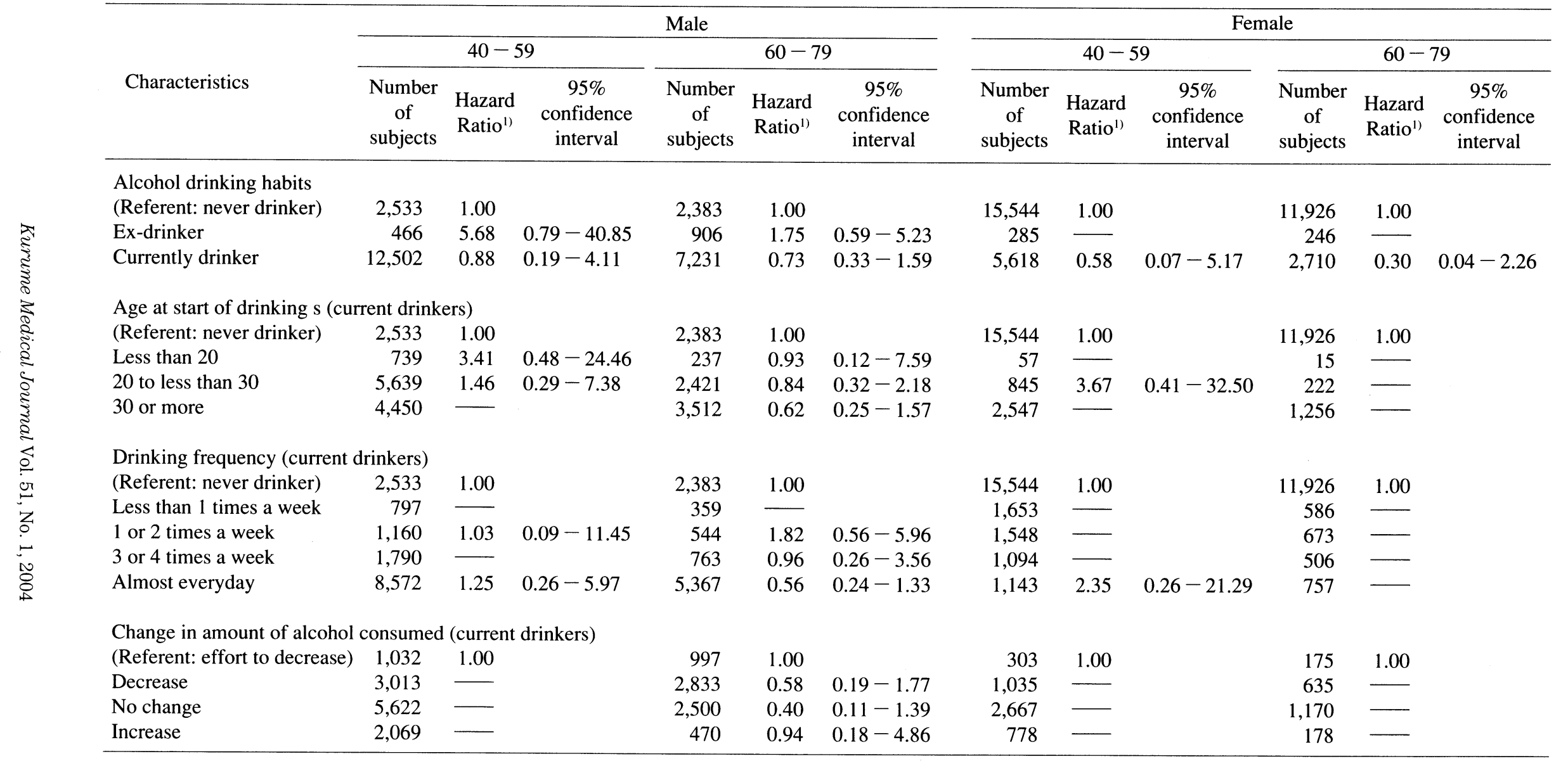

1) Result from SAS PHREG procedure with STRATA statement on variable concerning with collaborating institute.

${ }^{*} \mathrm{p}<0.05, \wedge \mathrm{p}<0.10$

- HR is not available because of thin data. 
TABLE 4.

Hazard ratio of death from HCC by drinking amount among total current drinkers

\begin{tabular}{|c|c|c|c|c|c|c|c|c|c|c|c|c|}
\hline \multirow[b]{3}{*}{ Characteristics } & \multicolumn{6}{|c|}{ Male } & \multicolumn{6}{|c|}{ Female (age group) } \\
\hline & \multicolumn{3}{|c|}{$40-59$} & \multicolumn{3}{|c|}{$60-79$} & \multicolumn{3}{|c|}{$40-59$} & \multicolumn{3}{|c|}{$60-79$} \\
\hline & $\begin{array}{l}\text { Number } \\
\text { of } \\
\text { subjects }\end{array}$ & $\begin{array}{l}\text { Hazard } \\
\text { Ratio }^{1)}\end{array}$ & $\begin{array}{l}95 \% \\
\text { confidence } \\
\text { interval }\end{array}$ & $\begin{array}{l}\text { Number } \\
\text { of } \\
\text { subjects }\end{array}$ & $\begin{array}{l}\text { Hazard } \\
\text { Ratio }^{1)}\end{array}$ & $\begin{array}{l}95 \% \\
\text { confidence } \\
\text { interval }\end{array}$ & $\begin{array}{l}\text { Number } \\
\text { of } \\
\text { subjects }\end{array}$ & $\begin{array}{l}\text { Hazard } \\
\text { Ratio }^{1)}\end{array}$ & $\begin{array}{l}95 \% \\
\text { confidence } \\
\text { interval }\end{array}$ & $\begin{array}{l}\text { Number } \\
\text { of } \\
\text { subjects }\end{array}$ & $\begin{array}{l}\text { Hazard } \\
\text { Ratio }^{1)}\end{array}$ & $\begin{array}{l}95 \% \\
\text { confidence } \\
\text { interval }\end{array}$ \\
\hline $\begin{array}{l}\text { Amount of alcohol } \\
\text { consumed per occasion }\end{array}$ & & & & & & & & & & & & \\
\hline (Referent: never drinker) & 2,707 & 1.00 & & 2,550 & 1.00 & & 16,256 & 1.00 & & 12,816 & 1.00 & \\
\hline 3 gou or less & 9,175 & 0.57 & $0.23-1.41$ & 5,795 & 0.82 & $0.46-1.45$ & 1,989 & 0.66 & $0.08-5.11$ & 770 & 0.48 & $0.07-3.54$ \\
\hline More than 3 and up to 6 & 2138 & 080 & $011-727$ & 675 & - & & 08 & - & & 23 & - & \\
\hline $\begin{array}{l}\text { More than } 6 \text { and up to } 9 \\
\text { gou }\end{array}$ & 204 & - & $0.18 \quad 1.28$ & 46 & - & & 25 & - & & 0 & 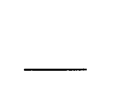 & \\
\hline $\begin{array}{l}\text { Cumulative amount of } \\
\text { alcohol consumed } \\
\text { (Referent: never drinker) }\end{array}$ & 2,707 & 1.00 & & 2,550 & 1.00 & & 16,256 & 1.00 & & 12,816 & 1.00 & \\
\hline Less than 50 gou $\cdot$ years & 6,535 & 0.39 & $0.13-1.17$ & 2,901 & 0.70 & $0.36-1.40$ & 1,519 & 0.91 & $0.12-7.08$ & 546 & 0.66 & $0.09-4.88$ \\
\hline $\begin{array}{l}50 \text { to less than } 100 \text { gou. } \\
\text { years }\end{array}$ & 3,446 & 0.92 & $0.33-2.56$ & 2,340 & 0.83 & $0.41-1.68$ & 110 & - & & 79 & - & \\
\hline $\begin{array}{l}100 \text { to less than } 150 \text { gou. } \\
\text { years } \\
150 \text { to less than } 200 \text { gou. }\end{array}$ & 453 & $\longrightarrow$ & & 585 & 1.49 & $0.54-4.06$ & 9 & - & & 13 & - & \\
\hline $\begin{array}{l}\text { years } \\
200 \text { gou } \cdot \text { years or more }\end{array}$ & $\begin{array}{r}76 \\
5\end{array}$ & - & & $\begin{array}{l}79 \\
30\end{array}$ & 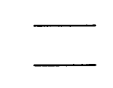 & & $\begin{array}{l}0 \\
0\end{array}$ & - & & $\begin{array}{l}0 \\
0\end{array}$ & - & \\
\hline
\end{tabular}

1) Result from SAS PHREG procedure with STRATA statement on variable concerning with collaborating institute.

${ }^{*} \mathrm{p}<0.05, \wedge \mathrm{p}<0.10$

HR is not available because of thin data. 
TABLE 5.

Hazard ratio of death from HCC by drinking amount among restricted current drinkers without past history of liver disease

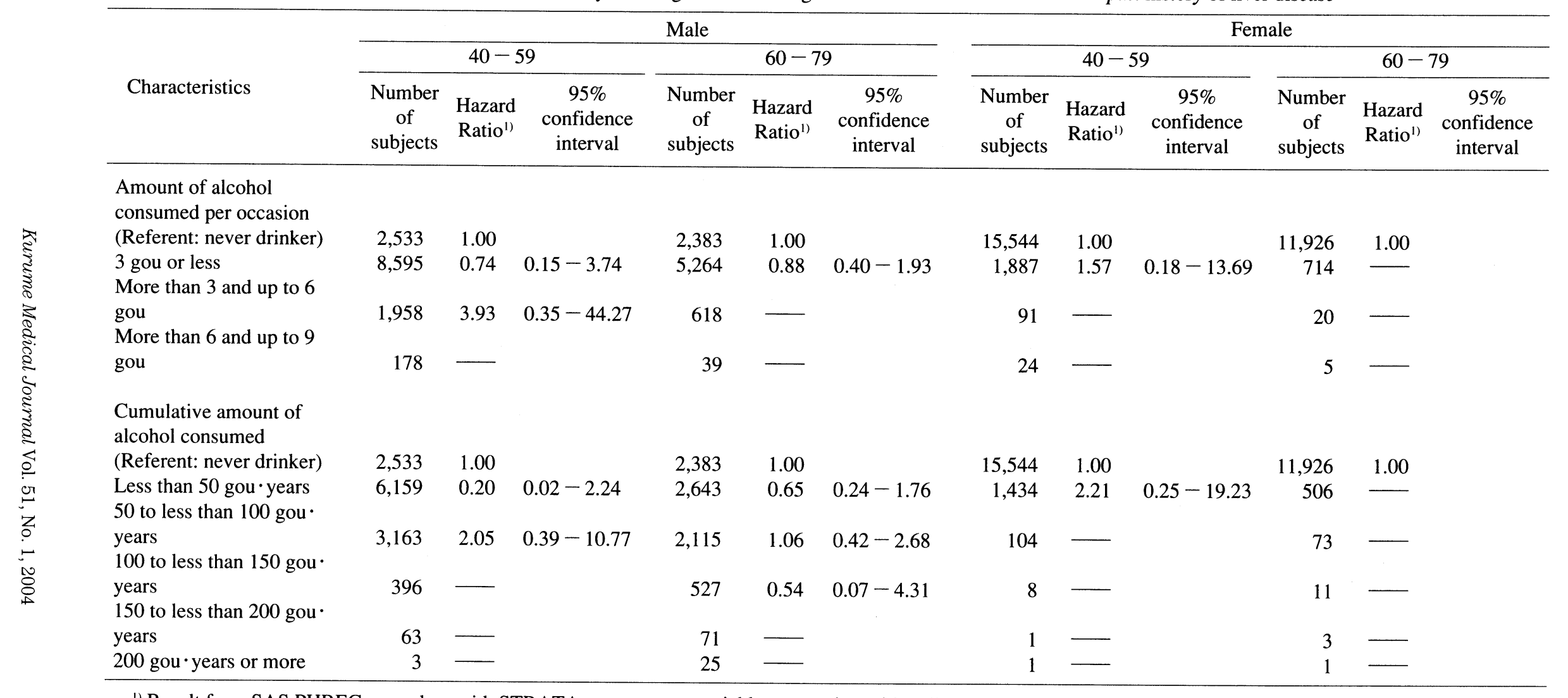

1) Result from SAS PHREG procedure with STRATA statement on variable concerning with collaborating institute.

${ }^{*} \mathrm{p}<0.05, \wedge \mathrm{p}<0.10$

HR is not available because of thin data. 
TABLE 6.

Hazard ratio of death from HCC by characteristics in alcohol drinking history of ex-drinkers and by summary drinking history among total subjects

\begin{tabular}{|c|c|c|c|c|c|c|c|c|c|c|c|c|}
\hline \multirow[b]{3}{*}{ Characteristics } & \multicolumn{6}{|c|}{ Male } & \multicolumn{6}{|c|}{ Female } \\
\hline & \multicolumn{3}{|c|}{$40-59$} & \multicolumn{3}{|c|}{$60-79$} & \multicolumn{3}{|c|}{$40-59$} & \multicolumn{3}{|c|}{$60-79$} \\
\hline & $\begin{array}{c}\text { Number } \\
\text { of } \\
\text { subjects }\end{array}$ & $\begin{array}{l}\text { Hazard } \\
\text { Ratio }^{1)}\end{array}$ & $\begin{array}{c}95 \% \\
\text { confidence } \\
\text { interval }\end{array}$ & $\begin{array}{c}\text { Number } \\
\text { of } \\
\text { subjects }\end{array}$ & $\begin{array}{l}\text { Hazard } \\
\text { Ratio }^{1)}\end{array}$ & $\begin{array}{c}95 \% \\
\text { confidence } \\
\text { interval }\end{array}$ & $\begin{array}{l}\text { Number } \\
\text { of } \\
\text { subjects }\end{array}$ & $\begin{array}{l}\text { Hazard } \\
\text { Ratio }^{1)}\end{array}$ & $\begin{array}{c}95 \% \\
\text { confidence } \\
\text { interval }\end{array}$ & $\begin{array}{c}\text { Number } \\
\text { of } \\
\text { subjects }\end{array}$ & $\begin{array}{l}\text { Hazard } \\
\text { Ratio }^{1)}\end{array}$ & $\begin{array}{c}95 \% \\
\text { d } \\
\begin{array}{c}\text { confidence } \\
\text { interval }\end{array}\end{array}$ \\
\hline \multicolumn{13}{|c|}{ Drinking frequency (ex-drinkers) } \\
\hline (Referent: never drinker) & 2,707 & 1.00 & & 2,550 & 1.00 & & 16,256 & 1.00 & & 12,816 & 1.00 & \\
\hline Less than 1 times a week & 69 & - & & 117 & 1.09 & $0.15-7.83$ & 78 & - & & 59 & 1.43 & $0.13-15.98$ \\
\hline 1 or 2 times a week & 63 & - & & 122 & 2.34 & $0.54-10.23$ & 67 & - & & 54 & 6.69 & $0.91-49.19^{\wedge}$ \\
\hline 3 or 4 times a week & 82 & 4.88 & $0.59-40.12$ & 123 & 4.23 & $1.24-14.46^{*}$ & 58 & - & & 46 & - & \\
\hline almost every day & 264 & 11.39 & $3.90-33.29^{*}$ & 460 & 4.78 & $2.34-9.75^{*}$ & 68 & - & & 67 & 4.76 & $0.65-35.01$ \\
\hline \multicolumn{13}{|c|}{ Age at cessation of drinking (ex-drinkers) } \\
\hline (Referent: never drinker) & 2,707 & 1.00 & & 2,550 & 1.00 & & 16,256 & 1.00 & & 12,816 & 1.00 & \\
\hline Less than 30 & 51 & - & & 25 & - & & 18 & - & & & & \\
\hline 30 to less than 45 & 180 & 4.86 & $1.01-23.47^{*}$ & 106 & 1.24 & $0.16-9.39$ & 129 & - & & 26 & 11.41 & $1.55-83.71^{*}$ \\
\hline 45 to less than 60 & 294 & 14.45 & $5.20-40.14^{*}$ & 308 & 4.83 & $2.13-10.91^{*}$ & 138 & 10.49 & $1.33-82.90^{*}$ & 88 & 6.46 & $1.53-27.35^{*}$ \\
\hline \multicolumn{13}{|c|}{ Years since cessation of drinking (ex-drinkers) } \\
\hline (Referent: never drinker) & 2,645 & 1.00 & & 2,496 & 1.00 & & 15,896 & 1.00 & & 12,562 & 1.00 & \\
\hline $0-5$ & 232 & 12.99 & $4.46-37.86^{*}$ & 393 & 1.32 & $0.39-4.52$ & 148 & 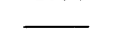 & & 81 & 3.79 & $0.51-27.86$ \\
\hline $6-10$ & 100 & 17.06 & $4.95-58.82^{*}$ & 210 & 4.90 & $2.02-11.87^{*}$ & 52 & 26.36 & $3.18-218.82^{*}$ & 46 & 6.57 & $0.89-48.55$ \\
\hline $11-15$ & 50 & - & & 122 & 5.73 & $1.92-17.13^{*}$ & 27 & - & & 34 & 7.87 & $1.06-58.58^{*}$ \\
\hline 16 or more & 94 & - & & 204 & 2.99 & $1.00-8.92^{*}$ & 36 & - & & 58 & 5.59 & $0.76-40.97$ \\
\hline \multicolumn{13}{|l|}{ Summary drinking history } \\
\hline $\begin{array}{l}\text { (Reterent: never arinker) } \\
\text { Current low frequency }\end{array}$ & $\begin{array}{r}2,101 \\
854\end{array}$ & $\begin{array}{l}1.00 \\
0.49\end{array}$ & $0.06-4.00$ & $\begin{array}{r}2,530 \\
398\end{array}$ & $\begin{array}{l}1.00 \\
0.98\end{array}$ & $0.29-3.35$ & $\begin{array}{r}1,250 \\
1,735\end{array}$ & 1.00 & & 638 & 0.92 & $0.22-3.86$ \\
\hline Low freq \& stopped early & 57 & -1 & & 48 & 2.65 & $0.35-19.97$ & 59 & 28.64 & $3.44-238.31^{*}$ & 21 & 8.77 & $1.15-66.75^{*}$ \\
\hline Low freq \& stopped lately & 4 & - & & 31 & - & & 8 & - & & 12 & 38.01 & $5.05-286.29^{*}$ \\
\hline Current frequent & 12,368 & 0.66 & $0.28-1.58$ & 7,381 & 0.71 & $0.40-1.25$ & 4,012 & 0.33 & $0.04-2.57$ & 2,144 & 0.27 & $0.06-1.13$ \\
\hline Frequent \& stopped early & 312 & 8.93 & $3.11-25.67^{*}$ & 146 & 5.56 & $2.18-14.15^{*}$ & 153 & - & & 44 & 7.85 & $1.07-57.67^{*}$ \\
\hline Frequent \& stopped lately & 53 & - & & 219 & 3.35 & $1.23-9.09^{*}$ & 18 & - & & 43 & 7.37 & $1.00-54.25^{*}$ \\
\hline
\end{tabular}

1) Result from SAS PHREG procedure with STRATA statement on variable concerning with collaborating institute.

$* \mathrm{p}<0.05, \wedge \mathrm{p}<0.10$

HR is not available because of thin data. 
TABLE 7.

Hazard ratio of death from HCC by characteristics in alcohol drinking history of ex-drinkers and by summary drinking history among restricted subjects without past history of liver disease

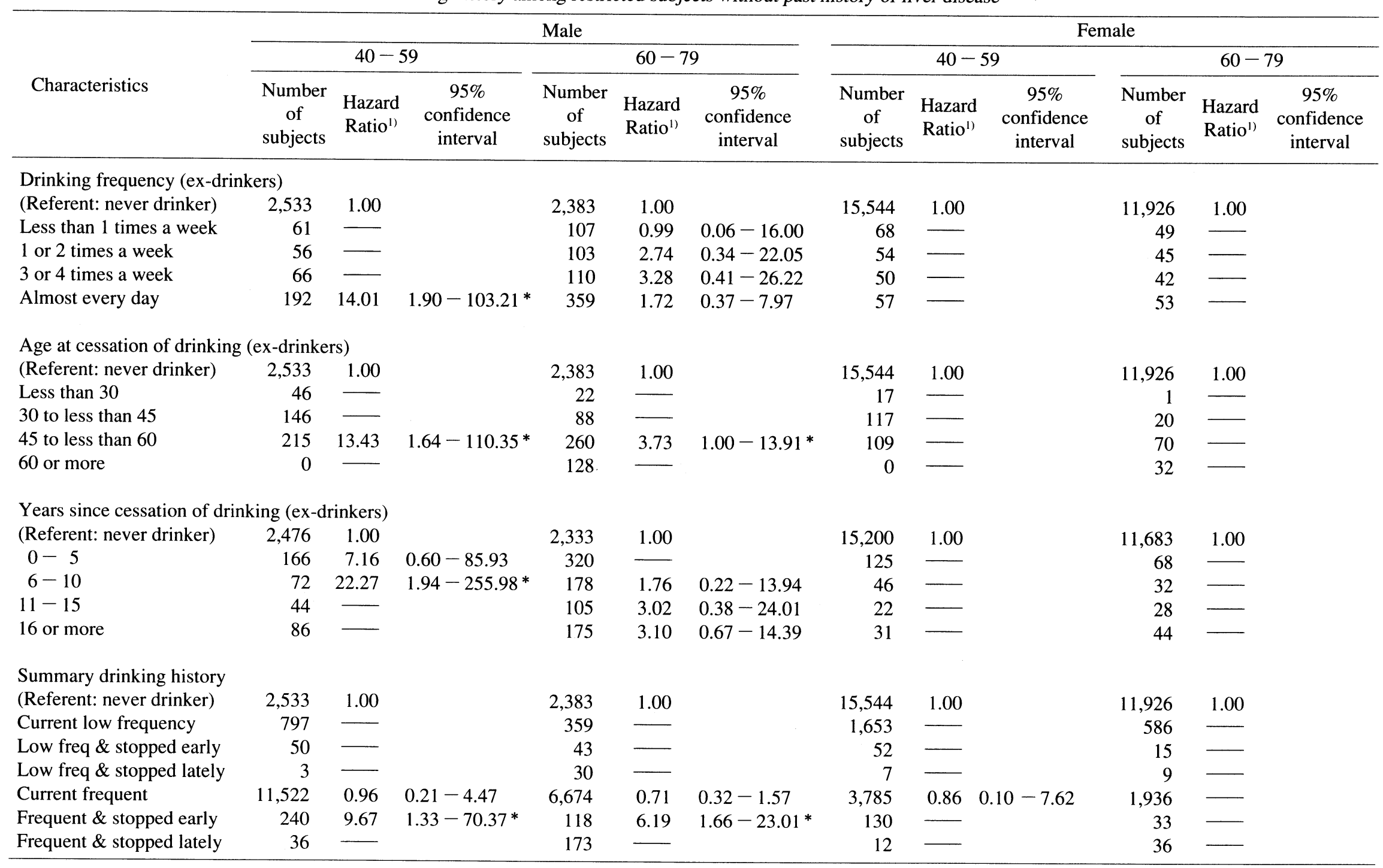

1) Result from SAS PHREG procedure with STRATA statement on variable concerning with collaborating institute.

${ }^{*} \mathrm{p}<0.05, \wedge \mathrm{p}<0.10$

- HR is not available because of thin data. 


\section{shown)}

According to the summary drinking history, among total subjects males who drank frequently and stopped early (before 50 years of age) had elevated HRs (Table 6,7). Besides this, older females who stopped drinking at age 30 or later had elevated HRs (Table 6).

\section{DISCUSSION}

In this study, the final outcome was defined as death from HCC, and confirmation of death from $\mathrm{HCC}$ was based on information that was considered highly reliable. On the other hand, information about exposure depended on responses to either self administered questionnaires or interviews, and such responses may not always be satisfactorily informative as regards drinking frequency for ex-drinkers, etc.

Many of the subjects of this study were participants in regional health check-up programs and they may therefore tend to have been healthier than average residents in their respective areas. But, as far as amount of alcohol consumed among current drinkers is concerned, no large difference was observed when compared to the national average [24].

In the univariate analysis of drinking habits, exdrinkers but not current drinkers were suggested to have a strong potential risk for death from HCC. Former studies have not pointed out such a discrepancy. Except for a few reports showing no or weak association [1,2], some cohort studies [3,4] and some case-control studies [5-14] showed evidence that current drinkers or heavy drinkers were exposed to the risk of HCC. Large cumulative alcohol intake was reported to be associated with increased risk in both these types of studies [7,16-19]. Unfortunately, JACC Study data lacked data on the amount of alcohol intake for ex-drinkers, so no direct evidence that ex-drinkers tended to have been heavy drinkers can be extracted from the data. However, the finding that a nearly daily drinking frequency had the highest and statistically significant HRs among total male exdrinkers may suggest this tendency. Also, the fact that males who continued drinking and gave it up at age 45 or later had increased HRs may also support this.

On the whole, LD was highly correlated to HCC (HR, male: 14.15, female: 27.72 ) in the previous study [21], and years since cessation of drinking showed a different association with HCC between subjects with or without LD in the present analysis.
These facts may support the hypothesis that exdrinkers might have suffered from some sort of serious hepatic dysfunction or symptom that forced them to quit drinking.

For younger male drinkers without LD, associations between HCC and several characteristics pertaining to drinking history, i.e. earlier age at start of drinking, larger amount of alcohol consumed per occasion, and larger cumulative consumption, were more strongly suggested than in the total subjects or those with LD. Large amount of consumption and frequent drinking are generally known to ruin one's liver or cause other physical ailments and such considerations might sometimes result in cessation of drinking. The strong association between ex-drinker status and HCC in this study may be a consequence of such drinking histories.

From these results, the pathway from beginning of alcohol use to HCC appeared to be complex, and more detailed multivariate analyses including confounders and interactions are needed to clarify the net association between alcohol drinking history and HCC.

ACKNOWLEDGMENTS: The authors wish to express their sincere appreciation to Dr. Kunio Aoki, Professor Emeritus, Nagoya University School of Medicine and the former chairman of the JACC Study Group, and also to Dr. Haruo Sugano, the former Director of the Cancer Institute of the Japanese Foundation for Cancer Research, who greatly contributed to the initiation of the study.

This work was supported by a Grant-in-Aid for Scientific Research on Priority Areas (2) (No. 14031222) from the Ministry of Education, Culture, Sports, Science and Technology of Japan. The JACC Study has also been supported by Grants-in-Aid for Scientific Research from the same ministry (Nos. 61010076, 62010074, 63010074, 1010068, $2151065,3151064,4151063,5151069,6279102$, and 11181101).

Japan Collaborative Cohort Study Group

The present members of the JACC Study and their affiliations are as follows: Dr. Akiko Tamakoshi (present chairman of the study group), Nagoya University Graduate School of Medicine; Dr. Mitsuru Mori, Sapporo Medical University School of Medicine; Dr. Yutaka Motohashi, Akita University School of Medicine; Dr. Ichiro Tsuji, Tohoku University Graduate School of Medicine; Dr. Yosikazu Nakamura, Jichi Medical School; Dr. Hiroyasu Iso, Institute of Community Medicine, University of Tsukuba; Dr. Haruo Mikami, Chiba Cancer Center; Dr. Yutaka Inaba, Juntendo University School of Medicine; Dr. Yoshiharu Hoshiyama, Showa University School of Medicine; Dr. Hiroshi Suzuki, Niigata University Graduate School of Medical and Dental Sciences; Dr. Hiroyuki Shimizu, Gifu University School of Medicine; Dr. Hideaki Toyoshima, Nagoya University Graduate School of Medicine; Dr. Shinkan Tokudome, Nagoya City University Graduate School of Medicine; Dr. Yoshinori 
Ito, Fujita Health University School of Health Sciences; Dr. Shuji Hashimoto, Fujita Health University School of Medicine; Dr. Shogo Kikuchi, Aichi Medical University School of Medicine; Dr. Akio Koizumi, Graduate School of Medicine and Faculty of Medicine, Kyoto University; Dr. Takashi Kawamura, Kyoto University Center for Student Health; Dr. Yoshiyuki Watanabe and Dr. Tsuneharu Miki, Kyoto Prefectural University of Medicine Graduate School of Medical Science; Dr. Chigusa Date, Faculty of Human Environmental Sciences, Mukogawa Women's University ; Dr. Kiyomi Sakata, Wakayama Medical University; Dr. Takayuki Nose, Tottori University Faculty of Medicine; Dr. Norihiko Hayakawa, Research Institute for Radiation Biology and Medicine, Hiroshima University; Dr. Takesumi Yoshimura, Institute of Industrial Ecological Sciences, University of Occupational and Environmental Health, Japan; Dr. Katsuhiro Fukuda, Kurume University School of Medicine; Dr. Naoyuki Okamoto, Kanagawa Cancer Center; Dr. Hideo Shio, Moriyama Municipal Hospital; Dr. Yoshiyuki Ohno (former chairman of the study group), Asahi Rosai Hospital; Dr. Tomoyuki Kitagawa, Cancer Institute of the Japanese Foundation for Cancer Research; Dr. Toshio Kuroki, Gifu University; and Dr. Kazuo Tajima, Aichi Cancer Center Research Institute.

The past investigators of the study group were listed in the ACKNOWLEDGEMENTS section [20] and the following seven past investigators were included as the present investigator in the list of MEMBERS OF JACC STUDY GROUP section [20]: Dr. Takashi Shimamoto, Dr. Heizo Tanaka, Dr. Shigeru Hisamichi, Dr. Masahiro Nakao, Dr. Takaichiro Suzuki, Dr. Tsutomu Hashimoto, and Dr. Teruo Ishibashi.

\section{REFERENCES}

1. Evans AA, Chen G, Ross EA, Shen FM, Lin WY et al. Eight-year follow-up of the 90,000-person Haimen City cohort: I. Hepatocellular carcinoma mortality, risk factors, and gender differences. Cancer-Epidemiol Biomarkers Prev 2002; 11:369-376.

2. Srivatanakul P, Parkin DM, Khlat M, Chenvidhya D, Chotiwan P et al. Liver cancer in Thailand. II. A casecontrol study of hepatocellular carcinoma. Int $\mathrm{J}$ Cancer 1991; 48:329-332.

3. Mukaiya M, Nishi M, Miyake H, and Hirata K. Chronic liver diseases for the risk of hepatocellular carcinoma: a case-control study in Japan. Etiologic association of alcohol consumption, cigarette smoking and the development of chronic liver diseases. Hepatogastroenterology 1998; 45:2328-2332.

4. Aizawa Y, Shibamoto Y, Takagi I, Zeniya M, and Toda G. Analysis of factors affecting the appearance of hepatocellular carcinoma in patients with chronic hepatitis $\mathrm{C}$. A long term follow-up study after histologic diagnosis. Cancer 2000; 89:53-59.

5. Fernandez E, La-Vecchia C, D’Avanzo B, Negri E, and Franceschi S. Family history and the risk of liver, gallbladder, and pancreatic cancer. Cancer Epidemiol
Biomarkers Prev 1994; 3:209-212.

6. Yu MW, Chiu YH, Chiang YC, Chen $\mathrm{CH}$, Lee TH et al. Plasma carotenoids, glutathione S-transferase M1 and T1 genetic polymorphisms, and risk of hepatocellular carcinoma: independent and interactive effects. Am J Epidemiol 1999; 149:621-629.

7. Tagger A, Donato F, Ribero ML, Chiesa R, Portera G et al. Case-control study on hepatitis $\mathrm{C}$ virus (HCV) as a risk factor for hepatocellular carcinoma: the role of $\mathrm{HCV}$ genotypes and the synergism with hepatitis B virus and alcohol. Brescia HCC Study. Int J Cancer 1999; 81:695699.

8. Tanaka K, Hirohata T, Takeshita S, Hirohata I, Koga S et al. Hepatitis B virus, cigarette smoking and alcohol consumption in the development of hepatocellular carcinoma: a case-control study in Fukuoka, Japan. Int J Cancer 1992; 51:509-514.

9. Mohamed AE, Kew MC, and Groeneveld HT. Alcohol consumption as a risk factor for hepatocellular carcinoma in urban southern African blacks. Int J Cancer 1992; 51:537-541.

10. Kuper H, Tzonou A, Kaklamani E, Hsieh CC, Lagiou P et al. Tobacco smoking, alcohol consumption and their interaction in the causation of hepatocellular carcinoma. Int J Cancer 2000; 85:498-502.

11. Chiba T, Matsuzaki Y, Abei M, Shoda J, Aikawa T et al. Multivariate analysis of risk factors for hepatocellular carcinoma in patients with hepatitis C virus-related liver cirrhosis. J Gastroenterol 1996; 31:552-558.

12. Tanaka K, Hirohata T, Fukuda K, Shibata A, Tsukuma H et al. Risk factors for hepatocellular carcinoma among Japanese women. Cancer Causes Control 1995; 6:91-98.

13. Hiyama T, Tsukuma H, Oshima A, and Fujimoto I. Liver cancer and life style-drinking habits and smoking habits. Gan No Rinsho 1990; 36:249-256. (in Japanese)

14. Tsukuma H, Hiyama T, Oshima A, Sobue T, Fujimoto I et al. A case-control study of hepatocellular carcinoma in Osaka, Japan. Int J Cancer 1990; 45:231-236.

15. Ikeda K, Saitoh S, Suzuki Y, Kobayashi M, Tsubota A et al. Disease progression and hepatocellular carcinogenesis in patients with chronic viral hepatitis: a prospective observation of 2215 patients. J Hepatol 1998; 28:930938.

16. Takeshita T, Yang X, Inoue Y, Sato S, and Morimoto K. Relationship between alcohol drinking, $\mathrm{ADH} 2$ and ALDH2 genotypes, and risk for hepatocellular carcinoma in Japanese. Cancer Lett 2000; 149:69-76.

17. Shibata A, Fukuda K, Nishiyori A, Ogimoto I, Sakata R et al. A case-control study on male hepatocellular carcinoma based on hospital and community controls. J Epidemiol 1998; 8:1-5.

18. Fukuda K, Shibata A, Hirohata I, Tanikawa K, Yamaguchi $\mathrm{G}$ et al. A hospital-based case-control study on hepatocellular carcinoma in Fukuoka and Saga Prefectures, Northern Kyushu, Japan. Jpn J Cancer Res 1993; 84:708-714.

19. Tanaka K, Hirohata T, and Takeshita S. Case-control studies of hepatocellular carcinoma and liver cirrhosis in relation to life style and other risk factors. Gan No Rinsho 1990; 36:305-312. (in Japanese) 
20. Ohno Y, Tamakoshi A, and the JACC Study Group. Japan Collaborative Cohort Study for Evaluation of Cancer Risk Spnsored by Monbusho (JACC Study). Journal of Epidemiology 2001; 11:144-150.

21. Shibata A, Ogimoto I, Kurozawa Y, Nose T, Yoshimura $\mathrm{T}$ et al. Past medical history and risk of death due to hepatocellular carcinoma. Kurume Medical Journal 2003; 50:109-119.

22. Research Group on Evaluation of Risk Factor for Cancer by Large-Scale Cohort Study, Baseline Result of A Large-Scale Cohort Study on Evaluation of Risk Factors on Cancer, 1996.

23. SAS Institute. The PHREG Procedure. In: SAS Procedures Guide, Version 8, ed. Sawyer DM and Stroke ME, SAS Institute Inc., Cary, NC, Vol 3, pp2569-2657, 1999.

24. The Ministry of Health and Welfare, Health Service Bureau. Physical status. In: National Nutrition Survey, 1989, The Ministry of Health and Welfare, Health Service Bureau, Dai-ichi Shuppan, Tokyo, pp119-170, 1991. (in Japanese) 